\title{
Emergence of SARS-CoV-2 Spike Escape Mutation Q493R After Bamlanivimab/Etesevimab Treatment for COVID-19
}

\section{Daniele Focosi}

North-Western Tuscany Blood Bank, Pisa University Hospital, Pisa, Italy

\section{Federica Novazzi}

Laboratory of Microbiology, ASST Sette Laghi, Varese, Italy

\section{Angelo Genoni}

Department of Medicine and Surgery, University of Insubria, Varese, Italy

\section{Francesco Dentali}

Department of Medicine and Surgery, University of Insubria, Varese, Italy

\section{Daniela Dalla gasperina}

Department of Medicine and Surgery, University of Insubria, Varese, Italy

\section{Andreina Baj ( $\square$ andreina.baj@uninsubria.it)}

Department of Medicine and Surgery, University of Insubria, Varese, Italy https://orcid.org/0000-00030088-2712

\section{Fabrizio Maggi}

Department of Medicine and Surgery, University of Insubria, Varese, Italy

\section{Case Report}

Keywords: SARS-CoV-2, COVID-19, variant of interest, Spike, receptor-binding motif, Q493R

Posted Date: May 18th, 2021

DOI: https://doi.org/10.21203/rs.3.rs-524959/v2

License: (a) (1) This work is licensed under a Creative Commons Attribution 4.0 International License. Read Full License

Version of Record: A version of this preprint was published at Emerging Infectious Diseases on July 27th, 2021. See the published version at https://doi.org/10.3201/eid2710.211538. 


\section{Abstract}

We report here the first in vivo selection of a Spike mutation (Q493R) conferring simultaneoius full resistance to both bamlanivimab and etesivimab.

\section{Main Text}

Accelerated SARS-CoV-2 evolution occurs under selective pressure by therapeutic interventions with neutralizing antibodies ${ }^{1}$. Bamlanivimab has been recently withdrawn from the vendor as a monotherapy because of failure against E484K SARS-CoV-2 variants. Emergency use remains authorized for the bamlanivimab/etesevimab cocktail ${ }^{2}$, for which no completely resistant variant has been reported to date in vivo.

A 73-years old male was diagnosed on February 2021 with cholangiocarcinoma: while waiting for chemotherapy, he developed sepsis and was admitted to Varese hospital on Apr 12 for steroid and antimicrobial treatment. Nasopharyngeal swab (NPS) for SARS CoV-2 RT-PCR was negative on admission, but positive on Apr 24. Given he had recovered from sepsis, on Apr 25 he was moved to the COVID unit of the hospital. Falling within one of the frail patient categories for emergency use of antiSpike monoclonal antibodies approved by the Italian Drug Agency and being seronegative for anti-S1/S2 IgG (Diasorin, Italy), on Apr 26 the patient received an i.v. infusion of bamlanivimab $700 \mathrm{mg} /$ etesevimab $1400 \mathrm{mg}$ at the hospital. RT-PCR performed on the NPS collected before infusion was positive for SARSCoV-2, with a cycle threshold (Ct) of 12 (Alinity, Abbott). Follow-up NPS remained positive on Apr 28 (Ct 15) and May 3 (Ct 24); a chest CT scan on Apr 30 showed progression to interstitial pneumonia, and the patient was placed in noninvasive ventilation.

SARS CoV-2 RT-PCR on nasopharyngeal swab (NPS) was performed with Alinity platform (Abbott), and anti-S1/S2 IgG were measured using a chemiluminescent immunoassay (Diasorin). Sanger sequencing of Spike gene on the Apr 24 NPS revealed a PANGOLIN clade B.1.1.7 (NextStrain clade 20I/501Y.V1, GenBank sequence MZ157261), which was 94\% prevalent in Italy at that time: since May 3 we observed a secondary A1478G peak in the Sgene, corresponding to Q493R amino acid mutation, which became predominant as soon as May 8 (Ct 18; GenBank sequence MZ157275) (Fig. 1).

E484, F490, Q493, and S494 are the 4 amino acid residues within the Spike receptor-binding motif (RBM) that are known to be critical for bamlanivimab binding. Q493 is also among the many more RBM residues crucial for interactions with etesivimab. Q493R/K (which can be selected in vitro by bamlanivimab ${ }^{3}$ ) is to date the only mutation that causes resistance to both bamlanivimab and etesivimab. In pseudoviral neutralization assays Q493R reduces susceptibility to bamlanivimab by $>6,666$ folds, to etesevimab by 232 folds, and to the combination of both by $>100$ folds ${ }^{2}$; accordingly, in a flow cytometry competitive assay, Q493R reduces $\mathrm{IC}_{50}$ of $>100$ folds for bamlanivimab and 42 folds for etesivimab ${ }^{3,4}$. 
Q493 mutations increase binding affinity to ACE2 ${ }^{5}$, but further studies are needed to clarify whether such escape mutants are fit enough to spread and persist in humans.

\section{Declarations}

We declare we have no conflict of interest related to this manuscript.

\section{References}

1. Chen L, Zody MC, Mediavilla JR, et al. Emergence of multiple SARS-CoV-2 antibody escape variants in an immunocompromised host undergoing convalescent plasma treatment. 2021: 2021.04.08.21254791.

2. US Food and Drug Administration. Emergency Use Authorization (EUA) for Bamlanivimab $700 \mathrm{mg}$ and Etesevimab 1400 mg IV Administered Together Center for Drug Evaluation and Research (CDER) Review 2021.

3. European Medicines Agency. Assessment report Eli Lilly and Company Limited use of bamlanivimab and etesevimab for the treatment of COVID-19. 2021.

4. Starr TN, Greaney AJ, Dingens AS, Bloom JD. Complete map of SARS-CoV-2 RBD mutations that escape the monoclonal antibody LY-CoV555 and its cocktail with LY-CoV016. Cel Reports Medicine 2021; 2(4): 100255.

5. Ortega JT, Pujol FH, Jastrzebska B, Rangel HR. Mutations in the SARS-CoV-2 spike protein modulate the virus affinity to the human ACE2 receptor, an in silico analysis. EXCLI journal 2021; 20: 585-600.

\section{Figures}




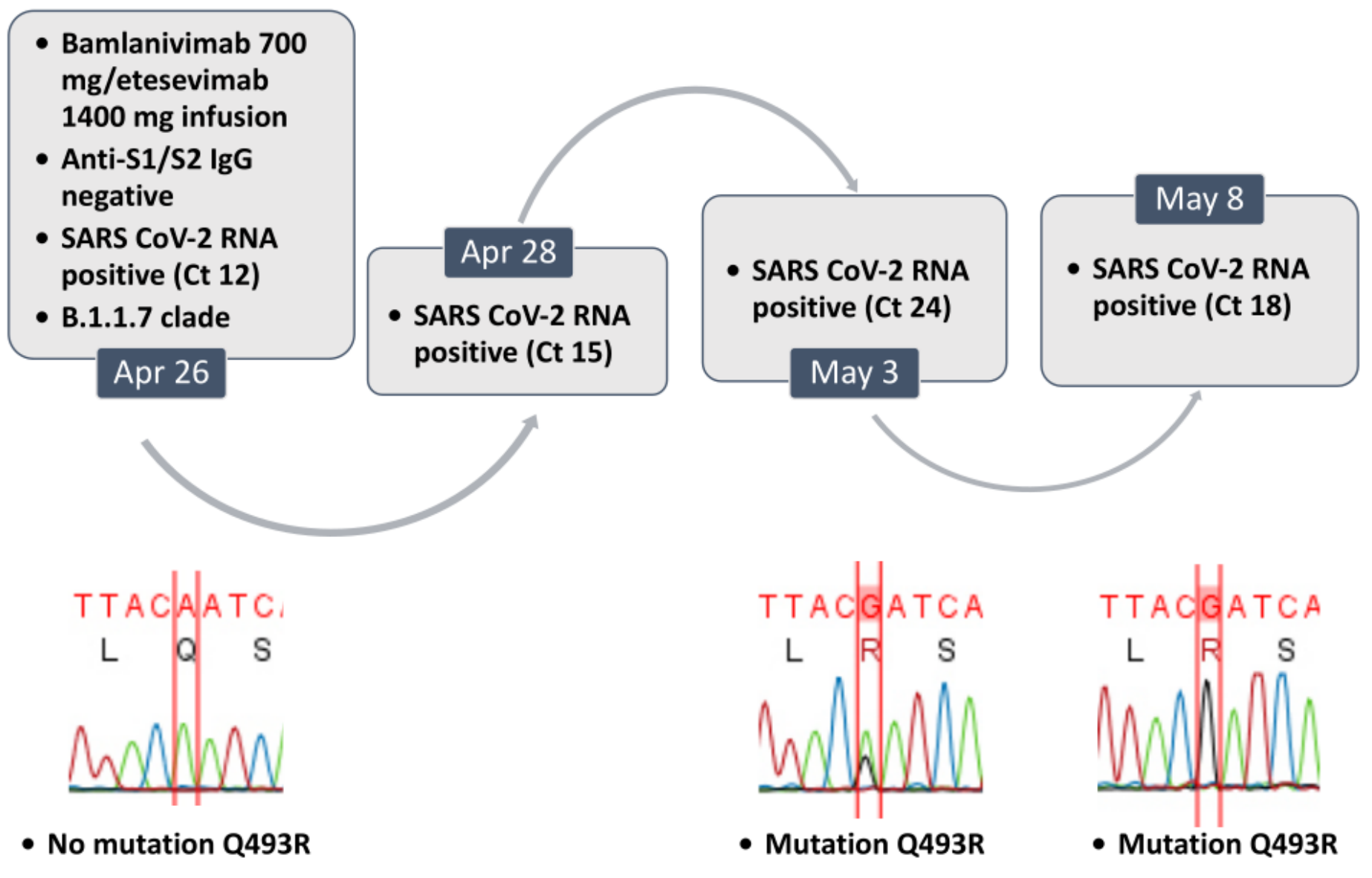

Figure 1

Evolution of SARS-CoV-2 variants in time after treatment with bamlanivimab-etesivimab. 